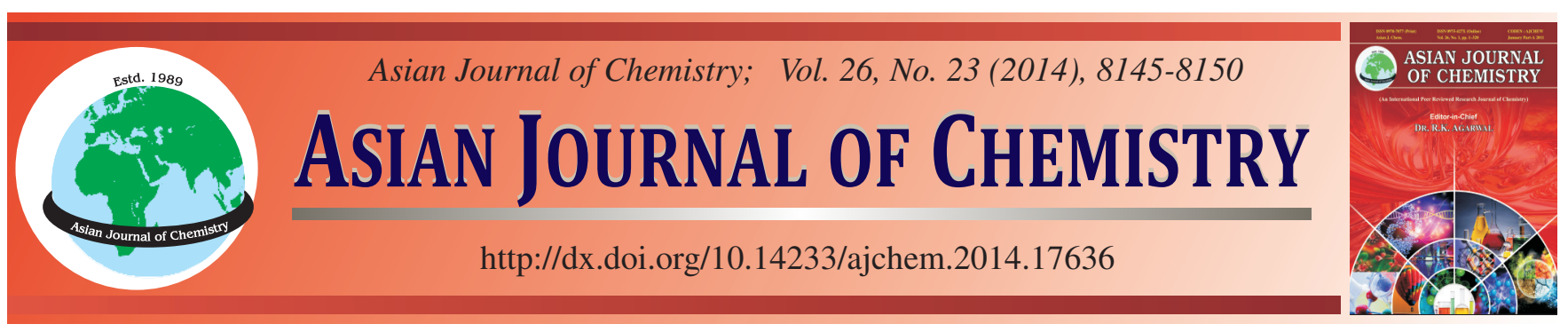

\title{
Synthesis and Characterization of bis-3,5-Disubstituted Thiadiazine-2-thione Derivatives as Anticancer Agents
}

\author{
A.A. Radwan ${ }^{1,2, *}$, T. Aboul-FadL ${ }^{3}$, A. Al-Dhfyan ${ }^{4,5}$ and W.M. Abdel-Mageeda ${ }^{6,7}$
}

\begin{abstract}
${ }^{1}$ Department of Pharmaceutical Organic Chemistry, Faculty of Pharmacy, Assiut University, Assiut 71526, Egypt
${ }^{2}$ Department of Pharmaceutics, College of Pharmacy, King Saud University, Riyadh 11451, Saudi Arabia

${ }^{3}$ Department of Medicinal Chemistry, Faculty of Pharmacy, Assiut University, Assiut 71526, Egypt

${ }^{4}$ Stem Cell Therapy Program, King Faisal Specialized Hospital and Research Center, Riyadh 11211, Saudi Arabia

${ }^{5}$ Department of Pharmacology, King Saud University, Riyadh, Saudi Arabia

${ }^{6}$ Pharmacognosy Department, College of Pharmacy, King Saud University, Riyadh 11451, P.O. Box 2457, Saudi Arabia

${ }^{7}$ Pharmacognosy Department, Faculty of Pharmacy, Assiut University, Assiut 71526, Egypt
\end{abstract}

*Corresponding author: Tel: +966146 70562; E-mail: dhna_2001@ hotmail.com

\begin{abstract}
Selective cytotoxic effect toward tumor cells but not the normal tissues represent a major challenge in cancer drug discovery. Herein, we describe the synthesis and selective antitumor activity of some HHT. All the synthesized compounds showed a good antitumor activity against leukemia K562 cells. In regard to cytotoxicity in normal cells, compounds (1-11) showed no cytotoxic effect within $200 \mu \mathrm{M}$ range, however compounds 12-16 showed non-selective cytotoxic effect.
\end{abstract}

Keywords: Synthesis, Thiadiazine-2-thiones, Anticancer.

\section{INTRODUCTION}

Cancer is a major public health concern worldwide. Transcending heart disease, cancer disease is the second leading cause of death due to different worldwide factors ${ }^{1-4}$. Cell division includes two following processes, mainly involves DNA copying and discrimination of replicated chromosomes into two separate cells. Initially, two stages are considered in cell division: mitosis (M), i.e. the process of nuclear split up and interphase i.e., the interval period between two $\mathrm{M}$ phases. The interphase stage constitutes G1, S and G2 phases through which the cells simply grow in size ${ }^{5}$. In the interphase stage, replication of DNA takes place in the $S$ phase which is preceded by G1 phase; a gap during which the cell prepares for DNA synthesis. Another, gap called G2 come after G1, during which the cell gets ready for mitosis. G1, S, G2 and M phases are the main parts of the standard cell cycle. Before involvement in DNA replication, cells in G1 enter a resting state called G0. Cells in G0 account for the major part of the non-growing, non-proliferating cells in the human body.

In cancer, cell proliferation results mainly from alterations in the genetic control of cell division. Two classes of genes are involved in this mutation including protooncogenes and tumour suppressor genes. In normal cells, the products of protooncogenes act at different levels along the pathways that stimulate cell proliferation ${ }^{6}$. Virus and inherited predisposing factors that contribute to mutated versions of protooncogenes or oncogenes can impair the G1 checkpoint function and promote tumour growth. During cytotoxic cancer treatment, more than half of all human cancer cells with impaired G1 function rely on the $\mathrm{G} 2$ to outrun the DNA damage. In normal cells, the G2 cell cycle is rarely utilized, which makes G2 checkpoint abrogation strategy attractive in cancer treatment ${ }^{7,8}$. Vinca alkaloids and taxanes are clinically used chemotherapeutics to control some types of cancer through their notable suppression of G2/M transition. The complex chemicalstructures of these compounds limit the possible structural modification and subsequently restrict the optimization of its anticancer activity. Thus, modulators of the G2/M checkpoint that have small molecular structures are of particular interest chemotherapeutic agents 9 .

Through our data from studies on the tetrahydro- $(2 H)$ 1,3,5-thiadiazine-2-thione (THTT) nucleus displays different biological activities ${ }^{6,10-16}$ including antitubercular, antibacterial, antiviral, antifungal, anthelmenthic and anticancer activities. The biological activity of these compounds was suggested based on isothiocyanates and dithiocarbamic acid species that are liberated in the biosystem upon hydrolysis ${ }^{17,18}$. Furthermore, 
effective in vivo anticancer activity have been reported with isothiocyanates obtained from cruciferous vegetables. They induce cell cycle arrest, which presupposes them as chemotherapeutic agents in cells with multidrug resistance phenotypes ${ }^{19,20}$. Recently, we had reported 3-cyclopentyl or (3-cyclohexyl)-5-substituted-3,4,5,6-tetrahydro- $2 \mathrm{H}$-1,3,5-thiadiazine2-thiones as a new class of molecules with active cell cycle inhibition activity ${ }^{4}$. These compounds, whose antiproliferative activity against cancer cells was promising, were synthesized through coupling of amino acids or their isosteres with the thiadiazine-2-thione skeleton.

In continuation of our work and in view of the findings suggesting that amino acid derivatives have a cell cycle arrest activity $^{21-23}$, we were prompted to synthesis of ethylene-bridged 1,3,5-thiadiazine-2-thiones incorporating amino acid in both of the connected thiadiazine moieties. The synthesized compounds were tested for their cell cycle inhibitory activity.

\section{EXPERIMENTAL}

The starting aminocarboxylic acids and all other chemicals were purchased from Sigma-Aldrich. Melting points were determined on an electrothermal melting point apparatus (Stuart Scientific, Stone) and were uncorrected. Precoated silica gel plates (kiesel gel $0.25 \mathrm{~mm}, 60 \mathrm{G} \mathrm{F} 254$, Merck) were used for thin layer chromatography (TLC). The developing solvent system was chloroform/methanol (9:1) and the spots were detected by ultraviolet light. Infrared (IR) spectra (KBr disc) were recorded on a FTIR spectrophotometer (Perkin Elmer) at the research center, College of Pharmacy, King Saud University, Saudi Arabia. ${ }^{1} \mathrm{H}$ Nuclear magnetic resonance (NMR) spectra were scanned in dimethylsulfoxide (DMSO)$d_{6}$ on a NMR spectrophotometer (Bruker AXS Inc.) operating at $500 \mathrm{MHz}$ for ${ }^{1} \mathrm{H}$ and $125.76 \mathrm{MHz}$ for ${ }^{13} \mathrm{C}$ at the aforementioned research center. Chemical shifts are expressed in dvalues (ppm) relative to tetramethylsilane (TMS) as an internal standard. Exchangeable protons were confirmed by addition of drop of $\mathrm{D}_{2} \mathrm{O}$. Mass spectra were taken on a model 320 MS spectrometer (Varian) at the research center, College of Pharmacy, King Saud University, Saudi Arabia. Mass spectral data are given as $m / z$ (Intensity \%). Elemental analyses were done on a model 2400 CHNSO analyzer (Perkin Elmer). Cytotoxicity investigation were determined at the Stem Cell Therapy Program, King Faisal Specialized Hospital and Research Center.

\section{General procedure}

Synthesis of new 3,3'-ethylenebis (5-substituted-1,3,5thiadiazine-2-thiones): Carbon disulfide (60 mmol, $3.6 \mathrm{~mL})$ was added quietly to a mixture of ethylene diamine $(10 \mathrm{mmol})$ and aqueous potassium hydroxide (40\%, $40 \mathrm{mmol}, 5.5 \mathrm{~mL})$. After stirring the reaction mixture at room temperature for $2 \mathrm{~h}$, formaldehyde solution ( $35 \%, 44 \mathrm{mmol}, 3.26 \mathrm{~mL}$ ) was added to the mixture and stirring was maintained for another $3 \mathrm{~h}$. To the resulting clear solution obtained, a solution of the appropriate amino acid $(20 \mathrm{mmol})$ in phosphate buffer $(\mathrm{pH}$ $7.8,20 \mathrm{~mL}$ ) was added slowly during $15 \mathrm{~min}$ and stirring was continued for $5 \mathrm{~h}$ at room temperature. Then, the reaction mixture was acidified with dilute hydrochloric acid (5\%) to $\mathrm{pH} 2$ and stirred for further $0.5 \mathrm{~h}$. The precipitate was collected by filtration, washed with $0.5 \%$ hydrochloric acid and dried. The crude solid was crystallized from ethanol to yield compounds 1-16.

5,5'-Ethylenebis [4-carbamoyl-2-(6-thioxo-1,3,5thiadiazinan-3-yl)butanoic acid] (1): Yield $3.2 \mathrm{~g}, 58 \%$, m.p. 143-144 ${ }^{\circ} \mathrm{C}$; IR (KBr, $\left.v_{\max }, \mathrm{cm}^{-1}\right)$ : 3419 (O-H carboxylic), 3341 (N-H amide), 2971 (C-H aliphatic), 1718 (C=O carboxylic), 1635 (C=O amide), $1491(\mathrm{C}=\mathrm{S}) ;{ }^{1} \mathrm{H}$ NMR (DMSO- $\left.d_{6}\right): 1.91$ (q, 2H, $\mathrm{HOOCCHCH}{ }_{2}-\mathrm{CH}_{2} \mathrm{CONH}_{2}$ ), 2.12 (t, 2H, HOOCCH$\mathrm{CH}_{2} \mathrm{CH}_{2} \mathrm{CONH}_{2}$ ), 3.43 (t, 1H, $\mathrm{HOOCCHCH} \mathrm{CH}_{2} \mathrm{CONH}_{2}$ ), 4.16 (s, $4 \mathrm{H}, \mathrm{NCH}_{2} \mathrm{CH}_{2} \mathrm{~N}$ ), 4.38 (s, 2H, $\left.\mathrm{NCH}_{2}-\mathrm{N}\right), 4.48$ (s, 2H, N-CH $2^{-}$ $\mathrm{S}$ ), 6.8 (s, exchangeable $2 \mathrm{H}, \mathrm{NH}_{2}$ ), 10.4 (br s, exchangeable $1 \mathrm{H}, \mathrm{CO}_{2} \mathrm{H}$ ); ${ }^{13} \mathrm{C}$ NMR (DMSO- $d_{6}$ ): 24.47 (butanoic C3), 30.34 (butanoic $\mathrm{C} 4$ ), 47.78 (ethylene $\mathrm{C} 1$ and $\mathrm{C} 2$ ), 55.6 (thiadiazine C-2), 60.85 (butanoic C2), 67.99 (thiadiazine C4), 173.16 (carboxylic $\mathrm{C}=\mathrm{O}$ ), 191.94 (thiadiazine $\mathrm{C}=\mathrm{S}$ ); $\mathrm{MS} \mathrm{m} / z$ [\%]: $220\left[\mathrm{M}^{+}-332,21\right], 205$ [100], 177 [13], 145 [16], 133 [19], 115 [20], 105 [25], 91 [30], 77 [19], 67 [12], 57 [63], 43 [17], 41 [56]. Anal. calcd. for $\mathrm{C}_{18} \mathrm{H}_{28} \mathrm{~N}_{6} \mathrm{O}_{6} \mathrm{~S}_{4}: \mathrm{C}, 39.11 ; \mathrm{H}, 5.11 ; \mathrm{N}$, 15.21; S, 23.21. Found: C, 39.36; H, 5.02; N, 15.13; S, 23.11.

5,5'-Ethylenebis [2-(6-thioxo-1,3,5-thiadiazinan-3yl)cyclopentanecarboxylic acid] (2): Yield 3.5 g, $67 \%$, m.p. 138- $139^{\circ} \mathrm{C}$; IR (KBr, $\left.\nu_{\max }, \mathrm{cm}^{-1}\right)$ : 3421 (O-H carboxylic), 2945 (C-H aliphatic), 1700 (C=O carboxylic), $1495(\mathrm{C}=\mathrm{S}) ;{ }^{1} \mathrm{H}$ NMR (DMSO- $\left.d_{6}\right): 1.65$ (m, 4H, two c-pentane-C4), 1.88 (m, 8H, two c-pentane $\mathrm{C} 3$ and $\mathrm{C} 5$ ), 3.02 (q, 2H, two c-pentane $\mathrm{C} 1$ ), 3.35 (q, 2H, two c-pentane $\mathrm{C} 2), 3.62\left(\mathrm{~s}, 4 \mathrm{H}, \mathrm{NCH}_{2} \mathrm{CH}_{2} \mathrm{~N}\right)$, 4.43 (s, 4H, two $\left.\mathrm{N}-\mathrm{CH}_{2}-\mathrm{N}\right), 4.69$ (s, 4H, two N-CH $\left.-\mathrm{S}\right), 10.76$ (br s, exchangeable $2 \mathrm{H}$, two $\mathrm{CO}_{2} \mathrm{H}$ ); ${ }^{13} \mathrm{C}$ NMR (DMSO- $d_{6}$ ): 21.54 (c-pentanyl C4), 26.64 (c-pentyl C5), 27.72 (c-pentyl C3), 43.95 (c-pentyl C1), 47.56 (ethylene C1 and C2), 51.89 (c-pentyl C2), 60.99 (thiadiazine C-2), 69.39 (thiadiazine C4), 175.34 (carboxylic $\mathrm{C}=\mathrm{O}$ ), 192.31 (thiadiazine $\mathrm{C}=\mathrm{S}$ ); $\mathrm{MS} \mathrm{m} / \mathrm{z}$ [\%]: $220\left[\mathrm{M}^{+}-298,22\right], 205$ [100], 177 [15], 145 [22], 133 [11], 115 [18], 105 [23], 91 [24], 77 [16], 67 [19], 57 [70], 43 [11], 41 [46]. Anal. calcd. for $\mathrm{C}_{20} \mathrm{H}_{30} \mathrm{~N}_{4} \mathrm{O}_{4} \mathrm{~S}_{4}$ : C, 46.31; H, 5.83; N, 10.80; S, 24.73. Found: C, 46.49; H, 5.71; N, 10.95; S, 24.62 .

5,5'-Ethylenebis [4-(6-thioxo-1,3,5-thiadiazinan-3yl)butanoic acid] (3): Yield $2.8 \mathrm{~g}, 60 \%$, m.p. $139-140{ }^{\circ} \mathrm{C}$; IR $\left(\mathrm{KBr}, v_{\max }, \mathrm{cm}^{-1}\right.$ ): 3435 (O-H carboxylic), 2939 (C-H aliphatic), 1715 (C=O carboxylic), $1494(\mathrm{C}=\mathrm{S}) ;{ }^{1} \mathrm{H}$ NMR (DMSO- $\left.d_{6}\right)$ : $1.76(\mathrm{~m}, 4 \mathrm{H}$, two butanoic $\mathrm{C} 3$ ), 2.28 ( $\mathrm{t}, 4 \mathrm{H}$, two butanoic $\mathrm{C} 2$ ), 2.72 (t, 4H, two butanoic C4), $4 . .17$ (s, 4H, $\left.\mathrm{NCH}_{2} \mathrm{CH}_{2} \mathrm{~N}\right), 4.5$ (s, 4H, two N-CH $-\mathrm{N}$ ), 4.59 (s, 4H, two N-CH $-\mathrm{S}$ ), 10.83 (br $\mathrm{s}$, exchangeable $2 \mathrm{H}$, two $\mathrm{CO}_{2} \mathrm{H}$ ); ${ }^{13} \mathrm{C}$ NMR (DMSO- $\left.d_{6}\right): 22.16$ (butanoic C3), 31.01 (butanoic C2), 47.47 (ethylene $\mathrm{C} 1$ and C2), 48.8 (butanoic C4), 57.58 (thiadiazine C-2), 70.02 (thiadiazine C4), 174.06 (carboxylic $\mathrm{C}=\mathrm{O}$ ), 191.84 (thiadiazine $\mathrm{C}=\mathrm{S}) ; \mathrm{MS} \mathrm{m} / z$ [\%]: 220 [M+246, 21], 205 [100], 177 [14], 145 [22], 133 [21], 115 [23], 105 [29], 91 [33], 77 [25], 67 [19], 57 [81], 43 [19], 41 [70]. Anal. calcd. for $\mathrm{C}_{16} \mathrm{H}_{26} \mathrm{~N}_{4} \mathrm{O}_{4} \mathrm{~S}_{4}$ : C, 41.18; H, 5.62; N, 12.01; S, 27.48. Found: C, 41.01; H, 5.73; N, 12.23; S, 27.25.

5,5'-Ethylenebis [4-(2-(6-thioxo-1,3,5-thiadiazinan-3yl)ethyl)benzoic acid] (4): Yield 3.6 g, $61 \%$, m.p. 141-142 ${ }^{\circ} \mathrm{C}$; IR (KBr, $v_{\max }, \mathrm{cm}^{-1}$ ): 3433 (O-H carboxylic), $3058(\mathrm{C}-\mathrm{H}$ aromatic), 2928 (C-H aliphatic), 1699 (C=O carboxylic), 1491 $(\mathrm{C}=\mathrm{S}), 938,751,668(\mathrm{ph}) ;{ }^{1} \mathrm{H}$ NMR $\left(\mathrm{DMSO}-d_{6}\right): 2.48(\mathrm{t}, 4 \mathrm{H}$, 
two $-\mathrm{CH}_{2} \underline{\mathrm{CH}}_{2}-\mathrm{Ph}$ ), 2.63 (t, $4 \mathrm{H}$, two $\left.-\mathrm{CH}_{2} \mathrm{CH}_{2}-\mathrm{Ph}\right), 4.15$ (s, 4H, $\left.\mathrm{NCH}_{2} \mathrm{CH}_{2} \mathrm{~N}\right), 4.53$ (s, $4 \mathrm{H}$, two N-CH$\left.-\mathrm{N}\right), 4.85$ (s, $4 \mathrm{H}$, two N$\mathrm{CH}_{2}-\mathrm{S}$ ), 10.68 (br s, exchangeable $2 \mathrm{H}$, two $\mathrm{CO}_{2} \mathrm{H}$ ); ${ }^{13} \mathrm{C} \mathrm{NMR}$ (DMSO- $\left.d_{6}\right)$ : $32.7\left(\mathrm{~N}-\mathrm{CH}_{2} \mathrm{CH}_{2}\right), 47.62$ (ethylene $\mathrm{C} 1$ and $\mathrm{C} 2$ ), $53.38\left(\mathrm{~N}-\mathrm{CH}_{2} \mathrm{CH}_{2}\right), 57.41$ (thiadiazine $\left.\mathrm{C}-2\right), 70.13$ (thiadiazine C4), 128.3 (Ar-C2 and Ar-C6), 129.01 (Ar-C3 and Ar-C5), 143.51 (Ar-C1), $167.8\left(\mathrm{CO}_{2} \mathrm{H}\right), 190.2(\mathrm{C}=\mathrm{S}) ; \mathrm{MS} \mathrm{m} / z$ [\%]: 220 [M+370, 28], 205 [100], 177 [16], 145 [19], 133 [14], 115 [24], 105 [25], 91 [18], 77 [22], 67 [16], 57 [65], 43 [21], 41 [58]. Anal. calcd. for $\mathrm{C}_{26} \mathrm{H}_{30} \mathrm{~N}_{4} \mathrm{O}_{4} \mathrm{~S}_{4}$ : C, 52.86; H, 5.12; N, 9.48; S, 21.71. Found: C, 52.95; H, 5.03; N, 9.31; S, 21.82.

5,5'-Ethylenebis[4-((6-thioxo-1,3,5-thiadiazinan-3yl)methyl)benzoic acid] (5): Yield 3.4 g, $60 \%$, m.p. 177-178 ${ }^{\circ} \mathrm{C}$; IR $\left(\mathrm{KBr}, \mathrm{v}_{\max }, \mathrm{cm}^{-1}\right)$ : 3421 (O-H carboxylic), $3042(\mathrm{C}-\mathrm{H}$ aromatic), 2933 (C-H aliphatic), 1693 (C=O carboxylic), 1486 $(\mathrm{C}=\mathrm{S}), 929,752,677(\mathrm{ph}) ;{ }^{1} \mathrm{H}$ NMR (DMSO- $\left.d_{6}\right)$ : $4.01(\mathrm{t}, 4 \mathrm{H}$, two $\left.-\underline{\mathrm{CH}_{2}}-\mathrm{Ph}\right), 4.18$ (s, $\left.4 \mathrm{H}, \mathrm{NCH}_{2} \mathrm{CH}_{2} \mathrm{~N}\right), 4.46$ (s, $4 \mathrm{H}$, two N$\mathrm{CH}_{2}-\mathrm{N}$ ), 4.61 (s, 4H, two N-CH $-\mathrm{S}$ ), 10.56 (br s, exchangeable $2 \mathrm{H}$, two $\mathrm{CO}_{2} \mathrm{H}$ ); ${ }^{13} \mathrm{C}$ NMR (DMSO- $d_{6}$ ): 47.72 (ethylene $\mathrm{C} 1$ and $\mathrm{C} 2), 53.38\left(\mathrm{~N}-\underline{\mathrm{CH}}_{2} \mathrm{Ph}\right), 57.36$ (thiadiazine $\left.\mathrm{C}-2\right), 69.94$ (thiadiazine C4), 128.7 (Ar-C2 and Ar-C6), 129.3 (Ar-C3 and Ar-C5), 141.92 (Ar-C1), $167.05\left(\mathrm{CO}_{2} \mathrm{H}\right), 191.8(\mathrm{C}=\mathrm{S})$; MS $m / z$ [\%]: $220\left[\mathrm{M}^{+}-342,19\right], 205$ [100], 177 [12], 145 [17], 133 [9], 115 [12], 105 [16], 91 [20], 77 [10], 67 [10], 57 [47], 43 [8]. Anal. calcd. for $\mathrm{C}_{24} \mathrm{H}_{26} \mathrm{~N}_{4} \mathrm{O}_{4} \mathrm{~S}_{4}: \mathrm{C}, 51.22 ; \mathrm{H}$, 4.66; N, 9.96; S, 22.79. Found: C, 51.44; H, 4.75; N, 9.82; S, 22.61 .

5,5'-Ethylenebis [5-(6-thioxo-1,3,5-thiadiazinan-3yl)pentanoic acid] (6): Yield $2.8 \mathrm{~g}, 59 \%$, m.p. $132-133{ }^{\circ} \mathrm{C}$; IR $\left(\mathrm{KBr}, v_{\max }, \mathrm{cm}^{-1}\right)$ : 3442 (O-H carboxylic), $2960(\mathrm{C}-\mathrm{H}$ aliphatic), 1716 ( $\mathrm{C}=\mathrm{O}$ carboxylic), $1490(\mathrm{C}=\mathrm{S}) ;{ }^{1} \mathrm{H}$ NMR (DMSO- $\left.d_{6}\right)$ : $1.48-1.66(\mathrm{~m}, 8 \mathrm{H}$, valeric acid C3, C4), 2.24 (t, $4 \mathrm{H}$, two valeric $\mathrm{C} 2), 2.48$ (t, $4 \mathrm{H}$, two valeric $\mathrm{C} 5), 3.86(\mathrm{~s}, 4 \mathrm{H}$, $\mathrm{NCH}_{2} \mathrm{CH}_{2} \mathrm{~N}$ ), 4.21 (s, $4 \mathrm{H}$, two $\left.\mathrm{N}_{-} \mathrm{CH}_{2}-\mathrm{N}\right), 4.61$ (s, 4H, two N$\mathrm{CH}_{2}-\mathrm{S}$ ), 10.74 (br s, exchangeable $2 \mathrm{H}$, two $\mathrm{CO}_{2} \mathrm{H}$ ); ${ }^{13} \mathrm{C} \mathrm{NMR}$ (DMSO- $d_{6}$ ): 21.34 (valeric C3), 27.81 (valeric C4), 33.01 (valeric C2), 47.47 (ethylene $\mathrm{C} 1$ and $\mathrm{C} 2$ ), 49.01 (valeric C5), 57.21 (thiadiazine C-2), 69.11 (thiadiazine C4), 173.18 (carboxylic $\mathrm{C}=\mathrm{O}$ ), 190.32 (thiadiazine $\mathrm{C}=\mathrm{S}$ ); $\mathrm{MS} \mathrm{m} / \mathrm{z}$ [\%]: 220 [M+274, 18], 205 [100], 177 [18], 145 [13], 133 [18], 115 [26], 105 [19], 91 [24], 77 [17], 67 [20], 57 [58], 43 [18], 41 [61]. Anal. calcd. for $\mathrm{C}_{18} \mathrm{H}_{30} \mathrm{~N}_{4} \mathrm{O}_{4} \mathrm{~S}_{4}$ : C, 43.70; H, 6.11; N, 11.33; S, 25.93. Found: C, 43.89; H, 6.03; N, 11.12; S, 25.74.

5,5'-Ethylenebis[4-((6-thioxo-1,3,5-thiadiazinan-3yl)methyl)cyclohexanecarboxylic acid] (7): Yield $3.8 \mathrm{~g}, 66$ \%, m.p. 141-142 ${ }^{\circ} \mathrm{C}$; IR (KBr, $\left.\mathrm{v}_{\max }, \mathrm{cm}^{-1}\right)$ : $3436(\mathrm{O}-\mathrm{H}$ carboxylic), 2982 (C-H aliphatic), 1708 (C=O carboxylic), $1495(\mathrm{C}=\mathrm{S}) ;{ }^{1} \mathrm{H}$ NMR (DMSO-d $): 1.28$ (q, 8H, two c-hexane C3, C5), 1.84 (q, 8H, two c-hexane C2, C6), 1.56 (m, 2H two c-hexane C4), 2.48 (t, 4H, two $\mathrm{N}-\mathrm{CH}_{2}-\mathrm{C}$ ), 2.58 (t, $2 \mathrm{H}$ two chexane $\mathrm{C} 1), 4.17\left(\mathrm{~s}, 4 \mathrm{H}, \mathrm{NCH}_{2} \mathrm{CH}_{2} \mathrm{~N}\right), 4.55$ (s, 4H, two N$\mathrm{CH}_{2}-\mathrm{N}$ ), 4.9 (s, 4H, two N-CH $2-\mathrm{S}$ ), 10.81 (br s, exchangeable $2 \mathrm{H}$, two $\mathrm{CO}_{2} \mathrm{H}$ ); ${ }^{13} \mathrm{C}$ NMR (DMSO- $d_{6}$ ): 23.97 (c-hexane $\mathrm{C} 2$, C6), 27.79 (c-hexane C3, C5), 34.73 (c-hexane C4), 44.03 (chexane $\mathrm{C} 1), 47.01$ (ethylene $\mathrm{C} 1$ and $\mathrm{C} 2), 53.32\left(\mathrm{~N}_{-} \mathrm{CH}_{2-}\right)$, 56.831 (thiadiazine $\mathrm{C}-2$ ), 70.35 (thiadiazine $\mathrm{C} 4$ ), 171.92 (carboxylic $\mathrm{C}=\mathrm{O}$ ), 192.01 (thiadiazine $\mathrm{C}=\mathrm{S}$ ); $\mathrm{MS} \mathrm{m/z} \mathrm{[ \% ]:} 220$ $\left[\mathrm{M}^{+}-354,28\right], 205$ [100], 177 [21], 145 [15], 133 [16], 115 [22], 105 [23], 91 [18], 77 [13], 67 [16], 57 [62], 43 [23], 41
[72]. Anal. calcd. for $\mathrm{C}_{24} \mathrm{H}_{38} \mathrm{~N}_{4} \mathrm{O}_{4} \mathrm{~S}_{4}: \mathrm{C}, 50.15 ; \mathrm{H}, 6.66 ; \mathrm{N}, 9.75$; S, 22.31. Found: C, 50.38; H, 6.51; N, 9.59; S, 22.18.

5,5'-Ethylenebis [2-D-3-methyl-2-(6-thioxo-1,3,5thiadiazinan-3-yl)butanoic acid] (8): Yield $3.1 \mathrm{~g}, 63 \%$, m.p. 195-196 ${ }^{\circ} \mathrm{C}$; Yield 2.8 g, $60 \%$, m.p. $139-140{ }^{\circ} \mathrm{C}$; IR (KBr, $\left.v_{\max }, \mathrm{cm}^{-1}\right): 3448$ (O-H carboxylic), 2966 (C-H aliphatic), 1723 (C=O carboxylic), $1496(\mathrm{C}=\mathrm{S}) ;{ }^{1} \mathrm{H}$ NMR (DMSO- $\left.d_{6}\right): 0.94$ (d, 12H, four $\mathrm{CH}_{3}$ ), 2.09 (m, 2H, two $\mathrm{CH}$ ), 3.18 (d, 2H two -N-CH-CO), 4.09 (s, 4H, $\left.\mathrm{NCH}_{2} \mathrm{CH}_{2} \mathrm{~N}\right), 4.46$ (s, 4H, two N$\mathrm{CH}_{2}-\mathrm{N}$ ), 4.65 (s, 4H, two N-CH$-\mathrm{S}$ ), 12.15 (br s, exchangeable $2 \mathrm{H}$, two $\left.\mathrm{CO}_{2} \mathrm{H}\right) ;{ }^{13} \mathrm{C}$ NMR (DMSO-d $): 18.1\left(\mathrm{CH}_{3}\right), 27.66$ (isopropyl $\mathrm{CH}$ ), 47.79 (ethylene $\mathrm{C} 1$ and $\mathrm{C} 2$ ), 57.21 (thiadiazine C-2), 67.74 (thiadiazine C4), 172.24 (carboxylic C=O), 191.78 (thiadiazine $\mathrm{C}=\mathrm{S}$ ); MS m/z [\%]: 220 [M+2 274,23$], 205$ [100], 177 [18], 145 [12], 133 [21], 115 [26], 105 [33], 91 [15], 77 [22], 67 [11], 57 [66], 43 [18], 41 [68]. Anal. calcd. for $\mathrm{C}_{18} \mathrm{H}_{30} \mathrm{~N}_{4} \mathrm{O}_{4} \mathrm{~S}_{4}: \mathrm{C}, 43.70 ; \mathrm{H}, 6.11 ; \mathrm{N}, 11.33 ; \mathrm{S}, 25.93$. Found: C, 43.57; H, 6.29; N, 11.18; S, 25.81.

5,5'-Ethylenebis[2-L-3-methyl-2-(6-thioxo-1,3,5thiadiazinan-3-yl)butanoic acid] (9): Yield 3.4 g, 69 \%, m.p. 178-179 ${ }^{\circ} \mathrm{C}$; IR (KBr, $\left.\nu_{\max }, \mathrm{cm}^{-1}\right)$ : 3447 (O-H carboxylic), 2962 (C-H aliphatic), 1717 (C=O carboxylic), $1490(\mathrm{C}=\mathrm{S}) ;{ }^{1} \mathrm{H}$ NMR (DMSO- $d_{6}$ ): 0.94 (d, 12H, four $\left.\mathrm{CH}_{3}\right), 2.07$ (m, 2H, two $\mathrm{CH}$ ), 3.22 (d, 2H two -N-CH-CO), 4.14 (s, 4H, $\mathrm{NCH}_{2} \mathrm{CH}_{2} \mathrm{~N}$ ), 4.44 (s, 4H, two $\mathrm{N}-\mathrm{CH}_{2}-\mathrm{N}$ ), 4.73 (s, 4H, two $\mathrm{N}_{-} \mathrm{CH}_{2}-\mathrm{S}$ ), 12.23 (br s, exchangeable $2 \mathrm{H}$, two $\left.\mathrm{CO}_{2} \mathrm{H}\right) ;{ }^{13} \mathrm{C}$ NMR (DMSO- $\left.d_{6}\right): 17.45$ $\left(\mathrm{CH}_{3}\right), 27.67$ (isopropyl $\mathrm{CH}$ ), 47.78 (ethylene $\mathrm{C} 1$ and $\mathrm{C} 2$ ), 56.17 (thiadiazine C-2), 67.79 (thiadiazine C4), 172.26 (carboxylic $\mathrm{C}=\mathrm{O}$ ), 191.79 (thiadiazine $\mathrm{C}=\mathrm{S}$ ); $\mathrm{MS} \mathrm{m} / \mathrm{z}$ [\%]: 220 $\left[\mathrm{M}^{+}-274,24\right], 205$ [100], 177 [20], 145 [11], 133 [18], 115 [25], 105 [31], 91 [12], 77 [20], 67 [15], 57 [48], 43 [22], 41 [62]. Anal. calcd. for $\mathrm{C}_{18} \mathrm{H}_{30} \mathrm{~N}_{4} \mathrm{O}_{4} \mathrm{~S}_{4}$ : C, 43.70; H, 6.11; N, 11.33; S, 25.93. Found: C, 43.62; H, 6.01; N, 11.24; S, 25.75.

5,5'-Ethylenebis[D-2-(6-thioxo-1,3,5-thiadiazinan-3yl)propanoic acid] (10): Yield 2.7 g, $61 \%$, m.p. 126-127 ${ }^{\circ} \mathrm{C}$; IR $\left(\mathrm{KBr}, v_{\max }, \mathrm{cm}^{-1}\right)$ : 3445 (O-H carboxylic), 2968 (C-H aliphatic), $1721\left(\mathrm{C}=\mathrm{O}\right.$ carboxylic), $1491(\mathrm{C}=\mathrm{S}) ;{ }^{1} \mathrm{H}$ NMR (DMSO-d $)_{6}$ ): $1.36\left(\mathrm{~d}, 6 \mathrm{H}\right.$, two $\left.\mathrm{CH}_{3}\right), 3.69$ (m, 2H, two $\mathrm{CH}$ ), 4.14 (s, $4 \mathrm{H}, \mathrm{NCH}_{2} \mathrm{CH}_{2} \mathrm{~N}$ ), 4.46 (s, 4H, two $\left.\mathrm{N}_{-} \mathrm{CH}_{2}-\mathrm{N}\right), 4.82$ (s, $4 \mathrm{H}$, two N-CH $2-\mathrm{S}$ ), 10.68 (br s, exchangeable $2 \mathrm{H}$, two $\mathrm{CO}_{2} \mathrm{H}$ ); ${ }^{13} \mathrm{C}$ NMR (DMSO- $\left.d_{6}\right)$ : $15.62\left(\mathrm{CH}_{3}\right), 47.67$ (ethylene $\mathrm{C} 1$ and $\mathrm{C} 2), 55.04$ (thiadiazine $\mathrm{C}-2), 56.3(\mathrm{CH}), 68.04$ (thiadiazine C4), 171.27 (carboxylic $\mathrm{C}=\mathrm{O}$ ), 191.92 (thiadiazine $\mathrm{C}=\mathrm{S}$ ); MS $m / z$ [\%]: 220 [M+-218, 32], 205 [100], 177 [23], 145 [18], 133 [15], 115 [31], 105 [35], 91 [18], 77 [19], 67 [12], 57 [55], 43 [25], 41 [58]. Anal. calcd. for $\mathrm{C}_{14} \mathrm{H}_{22} \mathrm{~N}_{4} \mathrm{O}_{4} \mathrm{~S}_{4}$ : C, 38.34; H, 5.06; N, 12.77; S, 29.24. Found: C, 38.51; H, 4.92; N, 12.63; S, 29.38 .

5,5'-Ethylenebis[4-methyl-2-(6-thioxo-1,3,5-thiadiazinan-3-yl)pentanoic acid] (11): Yield 3.6 g, $69 \%$, m.p. 179$180{ }^{\circ} \mathrm{C}$; IR ( $\left.\mathrm{KBr}, \mathrm{v}_{\max }, \mathrm{cm}^{-1}\right): 3449$ (O-H carboxylic), 2956 (C-H aliphatic), 1717 (C=O carboxylic), $1492(\mathrm{C}=\mathrm{S}) ;{ }^{1} \mathrm{H}$ NMR (DMSO-d $)_{6}$ : $0.89\left(\mathrm{~d}, 12 \mathrm{H}\right.$, four $\left.\mathrm{CH}_{3}\right), 1.53(\mathrm{~m}, 2 \mathrm{H}$, two isopropyl $\mathrm{CH}), 1.6\left(\mathrm{~m}, 4 \mathrm{H}\right.$, two $\left.\mathrm{CH}_{2}\right), 3.53(\mathrm{t}, 2 \mathrm{H}$, two N-CH$\mathrm{CO}), 4.16$ (s, $\left.4 \mathrm{H}, \mathrm{NCH}_{2} \mathrm{CH}_{2} \mathrm{~N}\right), 4.54$ (s, 4H, two $\left.\mathrm{N}-\mathrm{CH}_{2}-\mathrm{N}\right)$, 4.72 (s, $4 \mathrm{H}$, two $\mathrm{N}_{-} \mathrm{CH}_{2}-\mathrm{S}$ ), 11.89 (br s, exchangeable $2 \mathrm{H}$, two $\left.\mathrm{CO}_{2} \mathrm{H}\right) ;{ }^{13} \mathrm{C}$ NMR (DMSO- $\left.d_{6}\right): 22.98\left(\mathrm{CH}_{3}\right), 23.78$ (pentanoic C4), 38.55 (pentanoic C3), 47.54 (ethylene $\mathrm{C} 1$ and $\mathrm{C} 2$ ), 55.61 (thiadiazine C-2), $58.41(\mathrm{CH}), 60.68$ (pentanoic $\mathrm{C} 2$ ), 67.66 
(thiadiazine $\mathrm{C} 4$ ), 173.2 (carboxylic $\mathrm{C}=\mathrm{O}$ ), 192.0 (thiadiazine

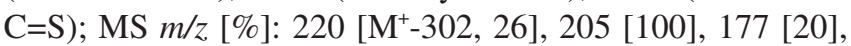
145 [15], 133 [11], 115 [22], 105 [32], 91 [16], 77 [14], 67 [11], 57 [61], 43 [33], 41 [67]. Anal. calcd. for $\mathrm{C}_{20} \mathrm{H}_{34} \mathrm{~N}_{4} \mathrm{O}_{4} \mathrm{~S}_{4}$ : C, 45.95; H, 6.56; N, 10.72; S, 24.53. Found: C, 45.82; H, $6.45 ; \mathrm{N}, 10.83 ; \mathrm{S}, 24.31$.

5,5'-Ethylenebis [2-(6-thioxo-1,3,5-thiadiazinan-3yl)ethanoic acid] (12): Yield 3.2 g, $78 \%$, m.p. $147-148{ }^{\circ} \mathrm{C}$; IR $\left(\mathrm{KBr}, v_{\max }, \mathrm{cm}^{-1}\right)$ : $3432(\mathrm{O}-\mathrm{H}$ carboxylic), $2944(\mathrm{C}-\mathrm{H}$ aliphatic), 1713 ( $\mathrm{C}=\mathrm{O}$ carboxylic), $1491(\mathrm{C}=\mathrm{S}) ;{ }^{1} \mathrm{H}$ NMR (DMSO-d $)_{6}$ : $0.89\left(\mathrm{~d}, 12 \mathrm{H}\right.$, four $\left.\mathrm{CH}_{3}\right), 3.57\left(\mathrm{~s}, 2 \mathrm{H}, \mathrm{N}-\mathrm{CH}_{2}-\right.$ CO), 4.17 (s, $4 \mathrm{H}, \mathrm{NCH}_{2} \mathrm{CH}_{2} \mathrm{~N}$ ), 4.53 (s, 4H, two $\mathrm{N}_{-} \mathrm{CH}_{2}-\mathrm{N}$ ), 4.61 (s, 4H, two $\mathrm{N}_{-} \mathrm{CH}_{2}-\mathrm{S}$ ), 11.3 (br s, exchangeable $2 \mathrm{H}$, two $\mathrm{CO}_{2} \mathrm{H}$ ); ${ }^{13} \mathrm{C}$ NMR (DMSO- $d_{6}$ ): 47.6 (ethylene $\mathrm{C} 1$ and $\mathrm{C} 2$ ), $50.81\left(\mathrm{~N}-\mathrm{CH}_{2}-\mathrm{CO}\right.$ (thiadiazine $\left.\mathrm{C}-2\right), 58.0$ (thiadiazine $\left.\mathrm{C}-2\right)$, 70.33 (thiadiazine C4), 170.39 (carboxylic $\mathrm{C}=\mathrm{O}$ ), 191.69 (thiadiazine $\mathrm{C}=\mathrm{S}$ ); MS m/z [\%]: 220 [M+1 190,32$], 205$ [100], 177 [22], 145 [16], 133 [14], 115 [25], 105 [27], 91 [12], 77 [18], 67 [18], 57 [45], 43 [26], 41 [58]. Anal. calcd. for $\mathrm{C}_{12} \mathrm{H}_{18} \mathrm{~N}_{4} \mathrm{O}_{4} \mathrm{~S}_{4}$ : C, 35.11; H, 4.42; N, 13.65; S, 31.24. Found: C, 35.04; H, 4.33; N, 13.52; S, 31.46.

5,5'-Ethylenebis[L-2-(6-thioxo-1,3,5-thiadiazinan-3yl)propanoic acid] (13): Yield 2.7 g, $61 \%$, m.p. $148-149{ }^{\circ} \mathrm{C}$; IR $\left(\mathrm{KBr}, \mathrm{v}_{\max }, \mathrm{cm}^{-1}\right)$ : $3426(\mathrm{O}-\mathrm{H}$ carboxylic), $2966(\mathrm{C}-\mathrm{H}$ aliphatic), 1716 (C=O carboxylic), $1494(\mathrm{C}=\mathrm{S}) ;{ }^{1} \mathrm{H}$ NMR (DMSO- $\left.d_{6}\right): 1.4\left(\mathrm{~d}, 6 \mathrm{H}\right.$, two $\left.\mathrm{CH}_{3}\right), 3.72(\mathrm{~m}, 2 \mathrm{H}$, two popanoic C2), 4.14 (s, 4H, $\mathrm{NCH}_{2} \mathrm{CH}_{2} \mathrm{~N}$ ), 4.48 (s, 4H, two $\mathrm{N}_{-} \mathrm{CH}_{2}-\mathrm{N}$ ), 4.87 (s, 4H, two $\mathrm{N}_{-} \mathrm{CH}_{2}-\mathrm{S}$ ), 10.83 (br s, exchangeable $2 \mathrm{H}$, two $\left.\mathrm{CO}_{2} \mathrm{H}\right) ;{ }^{13} \mathrm{C}$ NMR (DMSO- $\left.d_{6}\right): 15.67\left(\mathrm{CH}_{3}\right), 47.55$ (ethylene $\mathrm{C} 1$ and $\mathrm{C} 2$ ), 55.77 (thiadiazine $\mathrm{C}-2$ ), 56.33 (propanoic $\mathrm{C} 2$ ), 68.04 (thiadiazine C4), 173.42 (carboxylic C=O), 192.34 (thiadiazine $\mathrm{C}=\mathrm{S}$ ); $\mathrm{MS} \mathrm{m} / z$ [\%]: 220 [M+2 218,29$], 205$ [100], 177 [20], 145 [15], 133 [19], 115 [25], 105 [32], 91 [16], 77 [21], 67 [15], 57 [58], 43 [28], 41 [61]. Anal. calcd. for $\mathrm{C}_{14} \mathrm{H}_{22} \mathrm{~N}_{4} \mathrm{O}_{4} \mathrm{~S}_{4}$ : C, 38.34; H, 5.06; N, 12.77; S, 29.24. Found: C, 38.42; H, 5.18; N, 12.88; S, 29.41.

5,5'-Ethylenebis[3-(6-thioxo-1,3,5-thiadiazinan-3yl)propanoic acid] (14): Yield 2.6 g, $59 \%$, m.p. $144-145^{\circ} \mathrm{C}$; IR $\left(\mathrm{KBr}, \mathrm{v}_{\max }, \mathrm{cm}^{-1}\right)$ : 3421 (O-H carboxylic), $2927(\mathrm{C}-\mathrm{H}$ aliphatic), 1717 ( $\mathrm{C}=\mathrm{O}$ carboxylic), $1495(\mathrm{C}=\mathrm{S}) ;{ }^{1} \mathrm{H}$ NMR (DMSO-d $)$ : 2.52 (t, 4H, two CO-CH $\mathrm{CH}_{2}, 2.95(\mathrm{t}, 4 \mathrm{H}$, two N$\left.\mathrm{CH}_{2}\right), 4.20$ (s, 4H, $\left.\mathrm{NCH}_{2} \mathrm{CH}_{2} \mathrm{~N}\right), 4.51$ (s, 4H, two $\left.\mathrm{N}-\mathrm{CH}_{2}-\mathrm{N}\right)$, 4.58 (s, 4H, two $\mathrm{N}-\mathrm{CH}_{2}-\mathrm{S}$ ), 11.02 (br s, exchangeable $2 \mathrm{H}$, two $\mathrm{CO}_{2} \mathrm{H}$ ); ${ }^{13} \mathrm{C}$ NMR (DMSO- $d_{6}$ ): 36.25 (propanoic $\mathrm{C} 2$ ), 45.26 (propanoic C3), 47.23 (ethylene $\mathrm{C} 1$ and $\mathrm{C} 2$ ), 55.62 (thiadiazine C-2), 67.37 (thiadiazine C4), 172.51 (carboxylic $\mathrm{C}=\mathrm{O}$ ), 191.83 (thiadiazine $\mathrm{C}=\mathrm{S}$ ); MS $m / z$ [\%]: $220\left[\mathrm{M}^{+}-218,33\right], 205$ [100], 177 [18], 145 [19], 133 [23], 115 [24], 105 [25], 91 [19], 77 [33], 67 [12], 57 [59], 43 [27], 41 [74]. Anal. calcd. for $\mathrm{C}_{14} \mathrm{H}_{22} \mathrm{~N}_{4} \mathrm{O}_{4} \mathrm{~S}_{4}$ : C, 38.34; H, 5.06; N, 12.77; S, 29.24. Found: C, 38.22; H, 4.91; N, 12.64; S, 29.08.

5,5'-Ethylenebis[3-phenyl-2-(6-thioxo-1,3,5-thiadiazinan-3-yl)propanoic acid] (15): Yield 3.2 g, $54 \%$, m.p. 116$117{ }^{\circ} \mathrm{C}$; IR (KBr, $\left.v_{\max }, \mathrm{cm}^{-1}\right): 3420$ (O-H carboxylic), 2927 (C-H aliphatic), 1717 (C=O carboxylic), $1489(\mathrm{C}=\mathrm{S}) ;{ }^{1} \mathrm{H}$ NMR (DMSO-d $d_{6}$ : $3.1\left(\mathrm{~d}, 4 \mathrm{H}\right.$, two $\left.\mathrm{CH}_{2}\right), 3.81(\mathrm{t}, 2 \mathrm{H}$, two N-CHCO), 4.19 (s, 4H, $\mathrm{NCH}_{2} \mathrm{CH}_{2} \mathrm{~N}$ ), 4.62 (s, 4H, two $\mathrm{N}_{-} \mathrm{CH}_{2}-\mathrm{N}$ ), 4.78 (s, 4H, two $\mathrm{N}_{-} \mathrm{CH}_{2}-\mathrm{S}$ ), 7.28 (m, 10H, two $\mathrm{Ph}$ ), 10.86 (br s, exchangeable $2 \mathrm{H}$, two $\left.\mathrm{CO}_{2} \mathrm{H}\right) ;{ }^{13} \mathrm{C}$ NMR (DMSO- $\left.d_{6}\right): 36.25$ (propanoic C2), 45.26 (propanoic $\mathrm{C} 3$ ), 47.23 (ethylene $\mathrm{C} 1$ and C2), 55.62 (thiadiazine C-2), 67.37 (thiadiazine C4), 172.51 (carboxylic $\mathrm{C}=\mathrm{O}$ ), 191.83 (thiadiazine $\mathrm{C}=\mathrm{S}$ ); $\mathrm{MS} \mathrm{m} / \mathrm{z}$ [\%]: 220 [M+370, 33], 205 [100], 177 [24], 145 [18], 133 [23], 115 [31], 105 [29], 91 [21], 77 [32], 67 [21], 57 [54], 43 [39], 41 [68]. Anal. calcd. for $\mathrm{C}_{26} \mathrm{H}_{30} \mathrm{~N}_{4} \mathrm{O}_{4} \mathrm{~S}_{4}$ : C, 52.86; H, 5.12; N, 9.48; S, 21.71. Found: C, 52.93; H, 5.05; N, 9.61; S, 21.88.

5,5'-Ethylenebis[L-2-(6-thioxo-1,3,5-thiadiazinan-3yl)succinic acid] (16): Yield $2.5 \mathrm{~g}, 48 \%$, m.p. $164-165^{\circ} \mathrm{C}$; IR (KBr, $v_{\max }, \mathrm{cm}^{-1}$ ): 3448 (O-H carboxylic), 2923 (C-H aliphatic), 1721 ( $\mathrm{C}=\mathrm{O}$ carboxylic), $1491(\mathrm{C}=\mathrm{S}) ;{ }^{1} \mathrm{H}$ NMR (DMSO-d $)$ : 2.73 (d, 4H, two $\mathrm{CH}_{2}$ ), 3.83 (t, 2H, two $\mathrm{CH}$ ), 4.19 (s, 4H, $\mathrm{NCH}_{2} \mathrm{CH}_{2} \mathrm{~N}$ ), 4.48 (s, 4H, two N-CH $-\mathrm{N}$ ), 4.65 (s, 4H, two N$\mathrm{CH}_{2}-\mathrm{S}$ ), 10.58 (br s, exchangeable $2 \mathrm{H}$, two $\mathrm{CO}_{2} \mathrm{H}$ ); ${ }^{13} \mathrm{C}$ NMR (DMSO- $d_{6}$ ): 35.16 (succinic $\mathrm{CH}_{2}$ ), 48.06 (ethylene $\mathrm{C} 1$ and C2), 55.77 (thiadiazine C-2), 59.37 (succinic $\mathrm{CH}$ ), 68.43 (thiadiazine C4), 171.76 (carboxylic $\mathrm{C}=\mathrm{O}$ ), 191.87 (thiadiazine $\mathrm{C}=\mathrm{S}) ; \mathrm{MS} m / z$ [\%]: 220 [M+-306, 28], 205 [100], 177 [25], 145 [20], 133 [12], 115 [28], 105 [34], 91 [15], 77 [21], 67 [15], 57 [48], 43 [31], 41 [68]. Anal. calcd. for $\mathrm{C}_{16} \mathrm{H}_{22} \mathrm{~N}_{4} \mathrm{O}_{8} \mathrm{~S}_{4}$ : C, 36.49; H, 4.21; N, 10.64; S, 24.35. Found: C, 36.63; H, 4.38; N, 10.81; S, 24.17.

WST-1 cell proliferation assay: The MCF-12A cell line is a non-tumorigenic epithelial cell line obtained from tissue taken at reduction mammoplasty from a nulliparous patient having fibrocystic breast disease that contained focal areas of intraductal hyperplasia ${ }^{24}$. K562 chronic myelogenous leukemia cells were purchased from the American Type Culture Collection.

K562 Cells were maintained in RPMI 1640 (Sigma), supplemented with $10 \%$ FCS (Cambrex Bio Science), 100 $\mathrm{IU} / \mathrm{mL}$ penicillin, $100 \mathrm{mg} / \mathrm{mL}$ streptomycin and $2 \mathrm{mmol} / \mathrm{L} \mathrm{L}-$ glutamine (Sigma) and MCF-12 cells were cultured in DMEM/ F-12. Cells were seeded into 96 -well plates at $0.4 \times 10^{4} /$ well and incubated overnight. The medium was replaced with fresh one containing the desired concentrations of the compounds. After $48 \mathrm{~h}, 10 \mu \mathrm{L}$ of the WST-1 reagent was added to each well and the plates were reincubated for $4 \mathrm{~h}$ at $37^{\circ} \mathrm{C}$. The amount of formazan was quantified using ELISA reader at $450 \mathrm{~nm}$.

\section{RESULTS AND DISCUSSION}

The target compounds, 1-16 were synthesized by the reaction of ethylenediamine with carbon disulfide and potassium hydroxide to produce the corresponding dithiocarbamate potassium salts, which were not isolated. Cyclocondensation of dithiocarbamate intermediate was done using formaline and appropriate amino acid (Fig. 1 and Table-1). Structural elucidations of the synthesized compounds were performed on the bases of spectral and elemental methods of analyses and were consistent with the target structures. Generally, all the synthesised compounds ${ }^{1-16}$ showed carboxylic group at the range of $3449-3420$ and $1723-1693 \mathrm{~cm}^{-1}$ for the hydroxyl and carbonyl functionalities, respectively. Furthermore, the thione groups were shown at the range of 1496$1486 \mathrm{~cm}^{-1}$. ${ }^{1} \mathrm{H}$ NMR spectra of compounds $\mathbf{1 - 1 6}$ indicated the presence of a singlet at $\delta 3.62-4.2 \mathrm{ppm}$ which could be assigned to $>\mathrm{NCH}_{2} \mathrm{CH}_{2} \mathrm{~N}<$. Characteristic singlets appeared at $\delta 4.21$ - 


\begin{tabular}{|c|c|c|}
\hline \multicolumn{3}{|c|}{$\begin{array}{c}\text { TABLE-1 } \\
\text { CELL GROWTH INHIBITORY ACTIVITY } \\
\text { OF THE SYNTHESIZED COMPOUNDS } 1-16\end{array}$} \\
\hline \multirow{2}{*}{ Compound no. } & \multicolumn{2}{|c|}{$\mathrm{IC} 0(\mu \mathrm{M}) \pm \mathrm{SEM}^{\mathrm{a}}$} \\
\hline & K562 & MCF12 \\
\hline 1 & $14.26 \pm 0.002$ & $\mathrm{NA}^{\mathrm{b}}$ \\
\hline 2 & $13.84 \pm 0.008$ & NA \\
\hline 3 & $12.9 \pm 0.006$ & $77.64 \pm 0.313$ \\
\hline 4 & $17.43 \pm 0.02$ & NA \\
\hline 5 & $40.74 \pm 0.162$ & NA \\
\hline 6 & $82.11 \pm 0.039$ & NA \\
\hline 7 & $28.49 \pm 0.083$ & NA \\
\hline 8 & $13.21 \pm 0.004$ & NA \\
\hline 9 & $12.93 \pm 0.006$ & NA \\
\hline 10 & $60.6 \pm 0.003$ & NA \\
\hline 11 & $13.18 \pm 0.019$ & NA \\
\hline 12 & $13.68 \pm 0.03$ & NA \\
\hline 13 & $16.16 \pm 0.097$ & $26 \pm 0.015$ \\
\hline 14 & $22.1 \pm 0.068$ & $31.74 \pm 0.014$ \\
\hline 15 & $23.96 \pm 0.41$ & $32.65 \pm 0.055$ \\
\hline 16 & $38.16 \pm 0.15$ & $28.35 \pm 0.026$ \\
\hline \multicolumn{3}{|c|}{$\begin{array}{l}\mathrm{IC}_{50} \text { : concentration of the compound }(\mu \mathrm{M}) \text { producing } 50 \% \text { cell } \\
\text { growth inhibition after } 48 \mathrm{~h} \text { of compound exposure, as determined } \\
\text { by the WST- } 1 \text { assay. Each experiment was run at least two times, } \\
\text { and the results are presented as average values } \pm \text { S.D.; }{ }^{b} \mathrm{NA} \text { : } \\
\text { Compounds having } \mathrm{IC}_{50} \text { value }>100 \mu \mathrm{M}\end{array}$} \\
\hline
\end{tabular}

Compounds having $\mathrm{IC}_{50}$ value $>100 \mu \mathrm{M}$
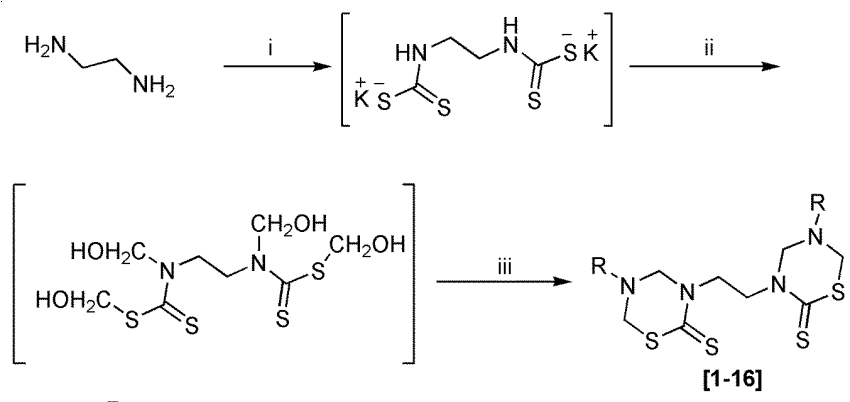

$\mathrm{R}=$

\begin{tabular}{|c|c|}
\hline $\begin{array}{l}\text { 1: } \mathrm{CH}(\mathrm{COOH}) \mathrm{CH}_{2} \mathrm{CH}_{2} \mathrm{CONH}_{2} \\
\text { 3: } \mathrm{CH}_{2} \mathrm{CH}_{2} \mathrm{CH}_{2} \mathrm{COOH} \\
5 ; \mathrm{CH}_{2} \mathrm{C}_{6} \mathrm{H}_{5} \mathrm{COOH}(\mathrm{p}) \\
\text { 7; } \mathrm{CH}_{2} \mathrm{cyclo}-\mathrm{C}_{6} \mathrm{H}_{10} \mathrm{COOH}(4) \\
\text { 9; } \mathrm{L}-\mathrm{CH}\left(\mathrm{CH}_{3}\right)_{2} \mathrm{CHCOOH} \\
11 ; \mathrm{L}^{-} \mathrm{CH}\left(\mathrm{CH}_{3}\right)_{2} \mathrm{CH}_{2} \mathrm{CHCOOH} \\
\text { 13; } \mathrm{L}-\mathrm{CH}_{3} \mathrm{CHCOOH} \\
\text { 15. }\end{array}$ & $\begin{array}{l}\text { 2; } \mathrm{cyClO}_{5} \mathrm{C}_{5} \mathrm{H}_{8} \mathrm{COOH}(2) \\
\text { 4; } \mathrm{CH}_{2} \mathrm{CH}_{2} \mathrm{C}_{6} \mathrm{H}_{5} \mathrm{COOH}(\mathrm{p}) \\
\text { 6; } \mathrm{CH}_{2} \mathrm{CH}_{2} \mathrm{CH}_{2} \mathrm{CH}_{2} \mathrm{COOH} \\
\text { 8; } \mathrm{D}-\mathrm{CH}\left(\mathrm{CH}_{3}\right)_{2} \mathrm{CHCOOH} \\
\text { 10; } \mathrm{D}-\mathrm{CH}_{3} \mathrm{CHCOOH} \\
\text { 12; } \mathrm{CH}_{2} \mathrm{COOH} \\
\text { 14; } \mathrm{CH}_{2} \mathrm{CH}_{2} \mathrm{COOH}\end{array}$ \\
\hline
\end{tabular}

Fig. 1. Synthetic pathway of compounds 1-16. Reagents and conditions: (i) $2 \mathrm{KOH}(20 \%), 2 \mathrm{CS}_{2}$, rt; (ii) $4 \mathrm{HCHO}$; (iii) $2 \mathrm{R}^{-} \mathrm{NH}_{2}$

4.62 and 4.9-4.48 ppm corresponding to $\mathrm{N}-\mathrm{CH}_{2}-\mathrm{N}$ and $\mathrm{N}-\mathrm{CH}_{2}-$ $\mathrm{S}$, respectively. ${ }^{13} \mathrm{C}$ NMR spectra of compounds $\mathbf{1 - 1 6}$ showed a characteristic signal at $\delta$ 47.01-48.06 ppm due to 3,3'ethhylene $\mathrm{C} 1$ and $\mathrm{C} 2$. Characteristic signals are shown at $\delta$ 50.81-60.99 and 67.37-70.35 ppm due to thiadiazine C-2 and thiadiazine $\mathrm{C} 4$, respectively. In addition, characteristic signals are shown at $\delta$ 167.05-175.34 and 190.2-192.34 ppm due to carboxylic carbonyl group and thione group, respectively. In the EI-Mass spectra, compounds 1-16 didn't show the molecular ion peak ${ }^{25}$ as it cleaved with formation of the stable cation $(\mathrm{m} / \mathrm{z} 220)$ as the base peak. This suggested a cleavage of the substituent at position-3, loss of carbon disulfide then demethylation.

WST-1 cell proliferation assay: The general in vitro antitumor screening of the newly synthesized compounds was performed using WST-1 cell proliferation assay. It is main objective to develop new compounds that can selectively kill those cells with highest tumorigenic potential and can be tolerated very well by surrounding healthy cells. Many of unwated effects of anti cancer compounds are as a reason of lack of selectivity toward cancer cells rather than normal cells. Thus, it worthy to developing novel compounds targeting only cancer cells for a given organ which is invaluable as pharmacological probes and represents possible lead structure for future therapeutic agents. Generally all the synthesized compounds showed a good antitumor activity against leukemia K562 cells (Table-1). Compounds 1-4,8,9,11-13 showed significant antitumor activity against leukemia K562 cells ( $\mathrm{IC}_{50}$ $=12.69-17.43 \mu \mathrm{M})$ and compounds 5,7,14-16 showed intermediate activity $\left(\mathrm{IC}_{50}=22.1-40.74 \mu \mathrm{M}\right)$ while compounds 6, 10 showed lower activity $\left(\mathrm{IC}_{50}=60.6 \mu \mathrm{M}\right.$ and $82.11 \mu \mathrm{M}$, respectively). However the majority showed good activity with $\mathrm{IC}_{50}$ less than $18 \mu \mathrm{M}$, it is very important to address that compounds 1-12 showed selective cytotoxic effect against leukemia K562 cells while compounds $\mathbf{1 3 - 1 6}$ revealed non selective cytotoxic effect against leukemia K562 cells and against MCF12 normal cells.

\section{Conclusion}

A series of 16 compounds was synthesized and the molecules were evaluated against K562 leukemia cells and MCF12 normal cells. Interestingly, several of the compounds showed promising selective activity against leukemia K562 cells. Of particular interest, compounds $\mathbf{1 - 4 , 8 , 9 , 1 1 - 1 2}$ showed significant antitumor activity against leukemia K562 cells $\left(\mathrm{IC}_{50}=\right.$ 12.69-13.68 $\mu \mathrm{M}$ ) with non toxic effect against normal cells. Thus this study provides several promising lead molecules which could be used for further development against cancer cells, particularly leukemia cells.

\section{ACKNOWLEDGEMENTS}

The authors would like to extend their sincere appreciation to the Deanship of Scientific Research at King Saud University for its funding of this research through the research Group Project no. RGP-VPP-326.

\section{REFERENCES}

1. J.C. Medina, D. Roche, B. Shan, R.M. Learned, W.P. Frankmoelle, D.L. Clark, T. Rosen and J.C. Jaen, Bioorg. Med. Chem. Lett., 9, 1843 (1999).

2. H.-Y. Park Choo, M. Kim, S.K. Lee, S. Woong Kim and I. Kwon Chung, Bioorg. Med. Chem., 10, 517 (2002).

3. B.S.P. Sandor Eckhardt, Curr. Med. Chem. Anticancer Agents, 2, 419 (2002).

4. A.A. Radwan, F.K. Alanazi and A. Al-Dhfyan, Drug Res., 63, 129 (2013).

5. C. Norbury and P. Nurse, Annu. Rev. Biochem., 61, 441 (1992).

6. A.A. Radwan, A. Al-Dhfyan, M.K. Abdel-Hamid, A.A. Al-Badr and T. Aboul-Fadl, Arch. Pharm. Res., 35, 35 (2012).

7. H. Dixon and C.J. Norbury, Cell Cycle, 1, 362 (2002).

8. T. Kawabe, Mol. Cancer Ther., 3, 513 (2004).

9. J.S. Lazo and K. Tamura, J. Pharmacol. Exp. Ther., 296, 364 (2001).

10. T. Aboul-Fadl and A. El-Shorbagi, Eur. J. Med. Chem., 31, 165 (1996).

11. T. Aboul-Fadl and K. Hassanin, Pharmazie, 54, 244 (1999).

12. T. Aboul-Fadl and A.-R. Khalil, Arzneimittelforschung, 53, 526 (2003).

13. T. Aboul-Fadl, M.A. Hussein, A.-N. El-Shorbagi and A.-R. Khallil, Arch. Pharm. Pharm. Med. Chem, 335, 438 (2002).

14. T. Aboul-Fadl and M. Osman, Sci. Pharm., 70, 359 (2002). 
15. A. Hyallah and A.A. Radwan, Bull. Pharm. Sci., 30, 39 (2007).

16. A.A. Radwan and N.A. Hussein, Bull. Pharm. Sci., 29, 255 (2006).

17. W. Schade and A. Rieche, Arch. Pharm., 299, 589 (1966).

18. R. Pérez, H. Rodríguez, E. Pérez, M. Suárez, O. Reyes and L.J. González, A.L. de Cerain, O. Ezpelata, C. Pérez and C. Ocho, Arzneimittelforschung-Drug Res., 50, 854 (2000).

19. J. Jakubikova, Y. Bao and J. Sedlak, Anticancer Res., 25, 3375 (2005).

20. L. Mi, N. Gan, A. Cheema, S. Dakshanamurthy, X. Wang, D.C.H. Yang and F.-L. Chung, J. Biol. Chem., 284, 17039 (2009).
21. S. Karunakaran, N.S. Umapathy, M. Thangaraju, T. Hatanaka, S. Itagaki, D.H. Munn, P.D. Prasad and V. Ganapathy, Biochem. J., 414, 343 (2008). 22. X.M. Lu, H.W. Yi, J.L. Xu, Y. Sun, J.X. Li, S.X. Cao and Q.A. Xu, J. Pharm. Pharmacol., 59, 1087 (2007).

23. P. Rose, M. Whiteman, P.K. Moore and Y.Z. Zhu, Nat. Prod. Rep., 22, 351 (2005).

24. T.M. Paine, H.D. Soule, R.J. Pauley and P.J. Dawson, Int. J. Cancer, 50, 463 (1992)

25. E. Saglam, S. Sarac, E. Kilic, M. Özalp and M. Ertan, Turk. J. Pharm. Sci., 8, 159 (2011). 